\title{
Proximity effect in superconducting bilayers and multilayers
}

\author{
J. Aarts, J. Meiresonne, H. Sprey, and W. Maj* \\ Kamerlingh Onnes Laboratorium, Leiden University, P.O. Box 9506, 2300 RA Leiden, The Netherlands \\ P. Zagwijn \\ FOM-Institute for Atomic and Molecular Physics, Kruislaan 407, \\ 1098 SJ Amsterdam, The Netherlands \\ (Received 19 September 1989)
}

\begin{abstract}
Measurements of the superconducting transition temperature $T_{c}$ of bilayers and multilayers of $\mathrm{Mo}_{69} \mathrm{Si}_{31} / \mathrm{Mo}_{47} \mathrm{Si}_{53}$ are presented. For the multilayers the results can be described in terms of the de Gennes-Werthamer theory for the proximity effect, without adjustable parameters. For the bilayers it is shown that a depression of $T_{c}$ occurs which can be described by the effects of weak localization in the same way as is already known for single thin films.
\end{abstract}

\section{INTRODUCTION}

When a superconducting thin film is brought into contact with a normal metal, a lowering of the superconducting transition temperature $T_{c}$, due to the proximity effect, is well known to occur. Nevertheless, detailed comparisons between the theoretically expected and the experimentally observed changes in $T_{c}$ upon varying the layer thickness are relatively scarce. This is the case for superconducting-normal metal bilayers as well as for multilayers. For bilayers, a problem is that the small thickness of the film may invoke other mechanisms for $T_{c}$ suppression, which are then difficult to separate from the proximity effect. In multilayers this problem is avoided, but still effects other than the proximity effect often change $T_{c}$, making comparison with the theory difficult. For instance, in the multilayer system $\mathrm{Nb}-\mathrm{Zr}_{\mathbf{r}}{ }^{1}$ interface mixing results in a thin layer of composition $\mathrm{Nb}_{0.5} \mathrm{Zr}_{0.5}$, which has a superconducting transition temperature higher than either of the constituents $\mathrm{Nb}$ or $\mathrm{Zr}$. In the system Mo-V, ${ }^{2}$ strain in the Mo and $\mathrm{V}$ layers develops below $70 \AA$ and causes a discontinuous change in the behavior of $T_{c}$. Finally, in the archetypical system $\mathrm{Nb}-\mathrm{Cu}$, $T_{c}$ decreases monotonously as a function of layer thickness, but for agreement between experiment and theory an intrinsic decrease of $T_{c}$ of the individual $\mathrm{Nb}$ layers with decreasing layer thickness has to be assumed. ${ }^{3,4}$ The aim of the present work is twofold. First, we want to investigate the proximity effect in a multilayer system where no interfering effects occur. Second, we want to compare the behavior of the multilayers with that of bilayers in order to identify additional $T_{c}$-suppressing effects in the bilayers. In this respect our experiments resemble recently reported work of Missert and Beasly, ${ }^{5}$ who investigated the effect of disorder on superconductivity in ultrathin multilayers of amorphous $\mathrm{Mo}_{1-x} \mathrm{Ge}_{x}$ alloys. The system chosen for the experiments consists of alternating layers of two different superconductors. For the superconductors we used two different concentrations of the amorphous alloy $\mathrm{Mo}_{x} \mathrm{Si}_{1-x}$. This choice has several advantages. One is that the alloys can be readily sputtered and the amorphous structure avoids problems with changes or strains in the crystal structure. Then, $\mathrm{Mo}_{x} \mathrm{Si}_{1-x}$ forms a continuous solid solution between $x=0$ and $x=0.75$ while $T_{c}$ changes linearly in at least the region between $x=0.45$ $\left(T_{c}=1.5 \mathrm{~K}\right)$ and $x=0.70\left(T_{c}=7 \mathrm{~K}\right),{ }^{6}$ which allows for tuning of the $T_{c}$ of the constituents. Also, the problem of interface mixing is minimalized, since the $T_{c}$ of the possible interface layer lies in between the $T_{c}$ of the constituents.

\section{EXPERIMENTAL}

A number of bilayers and multilayers were prepared by dc sputtering in an Ar atmosphere. Substrates used were $\mathrm{Si}$ and sapphire which were kept near room temperature during deposition. The target consisted of a Si wafer on which small pieces of Mo metal were fixed. The two halves of the wafer contained different amounts of Mo and alternate layers of about equal thickness were sputtered by moving a shutter in front of the wafer, thereby exposing one half or the other half of the target. The starting concentrations for the two constituent layers were $x=0.69$ (called $s$ layer, $T_{c s}=6.26 \mathrm{~K}$ ) and $x=0.47$ (called $n$ layer, $T_{c n}=1.7 \mathrm{~K}$ ). The ratio $T_{c n} / T_{c s}$ was therefore 0.27 . Thick single layers were sputtered before and during the preparation of the series in order to measure the bulk properties of the materials and to check on concentration changes. Multilayers consisted of eleven single layers, the first and last being an $n$ layer. Samples were characterized by electron microprobe analysis for the Mo and $\mathrm{Si}$ concentrations. The thick layers, almost all of the bilayers and some of the multilayers were also characterized by Rutherford backscattering. This gave estimates for the concentrations and thicknesses of individual layers, which were used as a calibration for the thickness monitor inside the vacuum chamber. For some samples the thickness was also measured by means of a stylus. After calibration a thickness ratio $d_{s} / d_{n}$ of 1.1 was found. One complication encountered was a small change in the Mo concentration for two samples at the end of the sputtered series. This resulted in $T_{c}$ changes of about $10 \%$. Since the $T_{c}$ ratio remained 0.27 the results presented below are scaled on the proper value of $T_{c s}$. Transition tempera- 
tures were determined by measuring the resistivity transition with a four-point technique and using the 10-90\% criterion. For these measurements, samples were patterned by photolithography and etching.

\section{RESULTS AND DISCUSSION}

For an analysis of the results we use the de GennesWerthamer formalism, ${ }^{7}$ which is valid in the dirty limit and relates the $T_{c}$ of a bilayer to bulk parameters of the constituents. The position-dependent order parameter $\Delta(x)$ in the $s$ and $n$ layer can be written as

$$
\Delta_{s}(x)=\exp \left( \pm i k_{s} x\right), \Delta_{n}(x)=\exp \left( \pm k_{n} x\right)
$$

The wave number $k_{s, n}$ satisfies the expressions

$$
\begin{aligned}
& \ln \left(T_{c} / T_{c s}\right)=\psi\left(\frac{1}{2}\right)-\psi\left(\frac{1}{2}+\frac{1}{2} \xi_{s}^{2} k_{s}^{2} T_{c s} / T_{c}\right), \\
& \ln \left(T_{c} / T_{c n}\right)=\psi\left(\frac{1}{2}\right)-\psi\left(\frac{1}{2}-\frac{1}{2} \xi_{n}^{2} k_{n}^{2} T_{c n} / T_{c}\right),
\end{aligned}
$$

where $T_{c s, n}$ are the transition temperatures of the constituents and $T_{c}$ is transition temperature of the bilayer. The coherence length $\xi_{s, n}$ is given by

$$
\xi_{i}^{2}=\frac{\pi \hbar k_{B}}{6 e^{2} T_{c i} \rho_{i} \gamma_{i}} \quad(i=s, n),
$$

where $\rho_{i}$ and $\gamma_{i}$ are the normal-state resistivities and linear specific-heat coefficients. The transition temperature $T_{c}$ can be calculated by also taking account of the boundary conditions for $\Delta(x)$. These are ${ }^{8} d \Delta(x) / d x=0$ at the film-vacuum interface and $\Delta /(N V)$ (with $N$ the density of states and $V$ the attractive interaction between electrons) continuous at the bilayer interface. This leads to the equation

$$
\frac{k_{s}}{\rho_{s}} \tan \left(k_{s} d_{s}\right)=\frac{k_{n}}{\rho_{n}} \tanh \left(k_{n} d_{n}\right)
$$

Here, $d_{s, n}$ is the thickness of the $s, n$ layer in the bilayer. It should be remembered that for a bilayer the condition $d \Delta(x) / d x=0$ is fulfilled at the vacuum interfaces, whereas for a multilayer, due to the symmetry, this condition is also fulfilled in the middle of an $s$ or $n$ layer. This implies that if bilayer and multilayer are to have the same $T_{c}$, the individual layer thicknesses in the multilayer have to be twice those of the bilayer. The consequence of this will be investigated experimentally below. The parameters needed to calculate the change in $T_{c}$ as a function of a layer thickness from Eqs. (1)-(4) are $T_{c n, s}, \rho_{n, s}$, and the product $(\rho \gamma)_{n, s}$ and these are all experimentally accessible. In our case $(\rho \gamma)_{n, s}$ is calculated from measurements of the perpendicular critical field as a function of temperature, using the relation

$$
\rho \gamma=\left.\frac{k_{B} \pi^{3}}{12 e} \frac{-d B_{c 2}}{d T}\right|_{T=T_{c}} .
$$

The parameters derived from measurements on thick layers of the constituent materials are given in Table I. The critical fields in these materials are high and the coherence lengths correspondingly low. The $-d B_{c 2} / d T$ for the high Mo concentration differed by about $5 \%$ for different samples and is $10 \%$ lower than the literature value. $^{6}$ Again for different samples the $-d B_{c 2} / d T$ for the low Mo concentration differed by more than $15 \%$. The value in Table $I$ is an average which is still about $20 \%$ lower than the literature value. The reason for this discrepancy was not found. The $T_{c}$ as calculated from these parameters and scaled on $T_{c s}$ is shown in Fig. 1 both for bilayers $\left(T_{c}^{\mathrm{bi}}\right)$ and for multilayers $\left(T_{c}^{\mathrm{mu}}\right)$ and show a characteristic decrease around a thickness of a few times the coherence length. As a consequence of the symmetry argument mentioned above, $T_{c}^{\mathrm{bi}}$ lies above $T_{c}^{\mathrm{mu}}$. The results of the measurements are also given in Fig. 1. In case of the multilayers, the measurements follow the calculated curve down to about $150 \AA$, but below $150 \AA$ the deviations become appreciable. To make the difference in behavior for bilayers and multilayers more clear, the values of $T_{c}^{\mathrm{bi}}-T_{c}^{\mathrm{mu}}$, again scaled on $T_{c s}$, are plotted in Fig. 2 . The calculation shows that this difference can be appreciable and should reach a maximum of about $0.8 \mathrm{~K}$ around a thickness of $150 \AA$. The measurements, however, do not show this behavior. Above $100 \AA$ the $T_{c}$ of the bilayers is larger than the $T_{c}$ of the corresponding multilayers, as expected, although the difference is smaller than calculated in Fig. 2. Below $100 \AA$, however, the difference even becomes inverted, an observation which is in agreement with those of Missert and Beasly. ${ }^{5}$

In order to explain the apparent discrepancies between experiment and calculation, it should be noted that most samples are thin films. This is clearly so for all bilayers, but it is also the case for the smallest multilayers. For instance the multilayer with $d_{s}=36 \AA$ has a total thickness of $370 \AA$. Both experimentally and theoretically it is known that when superconducting films become thin (for disordered films: on the scale of the electron diffusion length) the effects of weak localization start to suppress the superconductivity. ${ }^{5,9,10}$ Specifically, it was shown by Graybeal and Beasly ${ }^{10}$ that in thin films of $\mathrm{Mo}_{79} \mathrm{Ge}_{21}$ (an alloy very similar to $\mathrm{Mo}_{x} \mathrm{Si}_{1-x}$ ) the bulk transition temperature $T_{c 0}$ decreases with decreasing film thickness in a manner which can be described by a theoretical expression of Maekawa and Fukuyama. We find that above 50 $\AA$ this depression $T_{c} / T_{c 0}$ is a linear function of the sheet

TABLE I. Parameters for the two constituent layers of $\mathrm{Mo}_{x} \mathrm{Si}_{1-x}$.

\begin{tabular}{llllcllr}
\hline \hline Layer & $x$ & $T_{c}$ & \multicolumn{2}{c}{} & $-d B_{c 2} / d T$ & $\xi$ \\
$(\mathrm{K})$ & $T_{c} / T_{c s}$ & $(\mu \Omega \mathrm{cm})$ & $\rho / \rho_{n}$ & $(\mathrm{~K})$ & $(\AA)$ \\
\hline$s$ & 69 & 6.95 & 1.0 & 179 & 0.70 & 1.96 & 33 \\
$n$ & 47 & 1.72 & 0.27 & 260 & 1.0 & 2.2 & 59 \\
\hline \hline
\end{tabular}




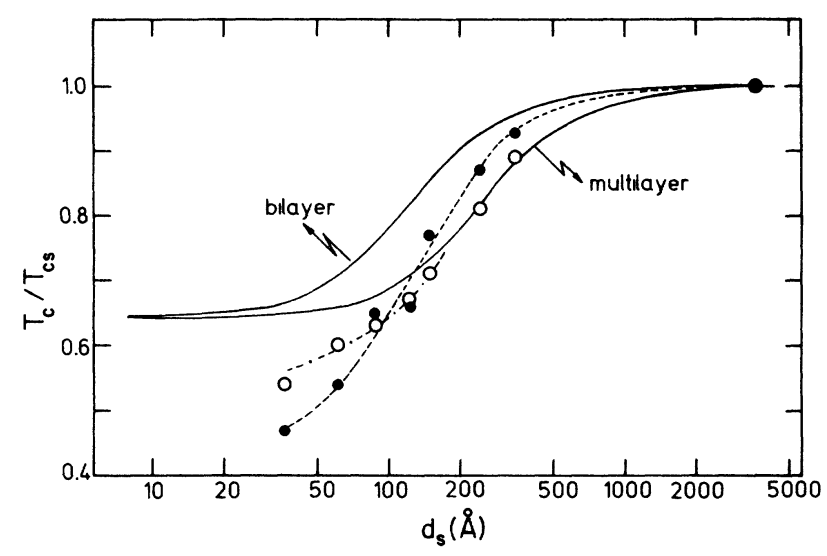

FIG. 1. $T_{c} / T_{c s}$ as a function of $d_{s}$ for bilayers and multilayers, calculated with the parameters of Table I. (O) Measurements on multilayers. (๑) Measurements on bilayers. Dashed and dashed-dotted lines are guides to the eye.

resistance $R_{\square}$,

$$
T_{c} / T_{c 0}=1-c R_{\square}(\Omega) / 100 .
$$

Here $c$ is a material dependent constant and is about 0.10 in the case of $\mathrm{Mo}_{79} \mathrm{Ge}_{21}$. ${ }^{9}$ In the case of $\mathrm{Mo}_{69} \mathrm{Si}_{31}$ we measured $T_{c} / T_{c 0}$ as function of $R_{\square}$ and find that the results are well described by $c=0.13$, very similar to $\mathrm{Mo}_{79} \mathrm{Ge}_{21}$. A simple way of correcting our results on bilayers and multilayers is now to assume that these act as single entities for the possible effects of localization. This assumption seems reasonable since the motion of the electrons is governed by a diffusion constant which is almost the same for both constituents. Also, because of the amorphous nature of the layers, extra effects of interface scattering are thought to be negligible. In Table II the values of $R_{\square}$ (derived from the measured resistance and the known length and width of the patterned samples) are given. It can be seen that also for the thinner multilayers the corrections are not negligible. In Fig. 3 the results are displayed again, but now corrected for localization effects using the inverse of Eq. (6). For the correction the value $c=0.11$

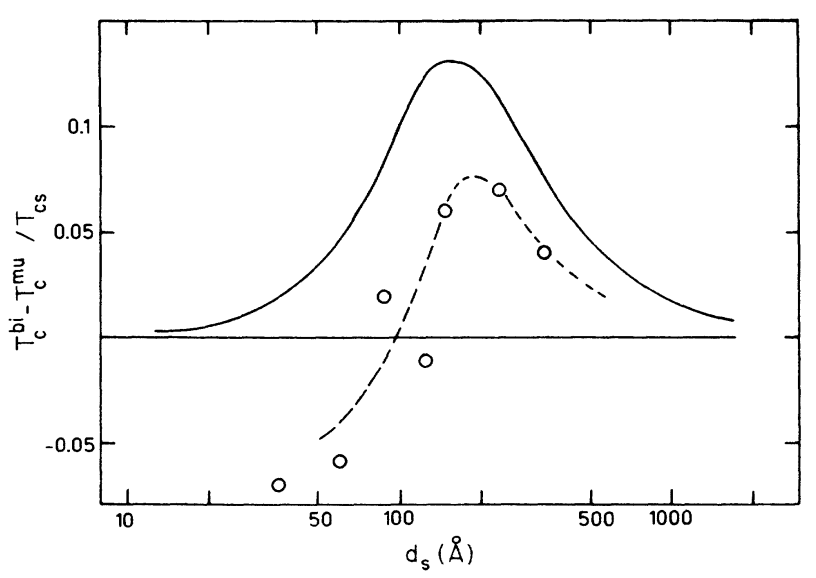

FIG. 2. Calculated difference $\left(T_{c}^{\mathrm{bi}}-T_{c}^{\mathrm{mu}}\right) / T_{c s}$ as a function of $d_{s}$ using the parameters in Table 1 . (O) Measurements. The dashed line is a guide to the eye.
TABLE II. Values of $R_{\square}$ for bilayers $\left(R_{\square}^{\mathrm{bi}}\right)$ and multilayers ( $R_{\square}^{\mathrm{mu}}$ ) with layer thickness $d_{s}$.

\begin{tabular}{lcrrrrrrr}
\hline \hline $\begin{array}{c}d_{s} \\
(\AA)\end{array}$ & $\begin{array}{c}R_{\mathrm{a}}^{\mathrm{bi}} \\
(\Omega)\end{array}$ & $\begin{array}{c}R_{\mathrm{a}}^{\mathrm{mu}} \\
(\Omega)\end{array}$ & $\begin{array}{c}d_{s} \\
(\AA)\end{array}$ & $\begin{array}{c}R_{\mathrm{ai}}^{\mathrm{bi}} \\
(\Omega)\end{array}$ & $\begin{array}{c}R_{\mathrm{au}}^{\mathrm{mu}} \\
(\Omega)\end{array}$ & $\begin{array}{c}d_{s} \\
(\AA)\end{array}$ & $\begin{array}{c}R_{\mathrm{a}}^{\mathrm{bi}} \\
(\Omega)\end{array}$ & $\begin{array}{c}R_{\mathrm{a}}^{\mathrm{mu}} \\
(\Omega)\end{array}$ \\
\hline 343 & 33 & 6 & 122 & 116 & 20 & 61 & 230 & 45 \\
243 & 57 & 10 & 88 & 160 & 29 & 36 & 480 & 78 \\
148 & 85 & 14 & & & & & & \\
\hline \hline
\end{tabular}

was used. There is no compelling reason to use the value of 0.13 found for single films of $\mathrm{Mo}_{69} \mathrm{Si}_{31}$ and $c-0.11$ was found to give the best agreement between measurements and calculations for the bilayers. The bilayer with $d_{s}=36$ $\AA$ is left out because its value of $R_{\square}$ is too high to be well described by Eq. (6). The correction proves very satisfactory, which appears to justify the assumption of singleentity behavior. The bilayers now completely follow the calculated behavior. For the multilayers the difference between measurements and calculation below $100 \AA$ is of the order of $3 \%$. This may be due to the slightly low value found for $-d B_{c 2} / d T$ of the normal-metal layer. In view of the experimental uncertainties it cannot be ascribed to the fact that the Werthamer theory does not well describe the (de Gennes-Cooper) limit $d_{s, n} \rightarrow 0,{ }^{11}$ especially since in the case of two superconductors the difference between the Werthamer calculation and the Cooper limit is probably less than in the case of a superconducting-normalmetal multilayer such as $\mathrm{Nb} / \mathrm{Cu} .^{4,11}$ An estimate for the Cooper limit using $N_{s}-N_{n}$ and a Debye temperature for MoSi of $300 \mathrm{~K}$ gives a value $T_{c} / T_{c s}=0.59$, only about $10 \%$ lower than the value from the Werthamer calculation.

In conclusion, we have shown that the change of transition temperature as a function of layer thickness in the multilayer system $\mathrm{Mo}_{69} \mathrm{Si}_{31} / \mathrm{Mo}_{47} \mathrm{Si}_{53}$ can be well described by the de Gennes-Werthamer theory for the proximity effect. Bilayers of the same system are also well described by the theory, but a correction has to be made for the effects of weak localization. These effects appear to affect the bilayers in much the same way as is already known for single thin films.

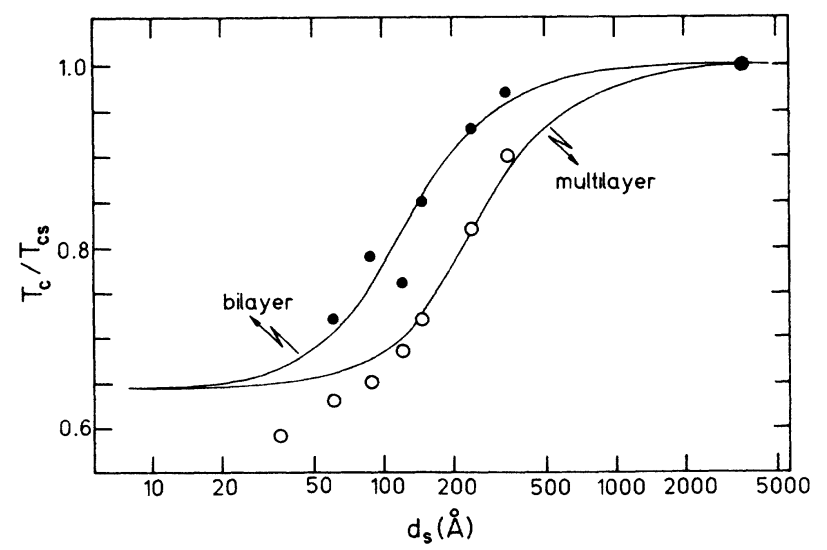

FIG. 3. $T_{c} / T_{c s}$ as a function of $d_{s}$ for bilayers and multilayers, calculated with the parameters of Table $I$. $(0,0)$ Measurements corrected for localization effects using $c=0.11$ (see text). 


\section{ACKNOWLEDGMENTS}

This work is part of the research program of the "Nederlandse Stichting voor Fundamenteel Onderzoek der Materie (FOM)." One of us (W.M.) also wishes to thank F.O.M. for financial support during his stay in Leiden. We are grateful for stimulating discussions with P. H. Kes and J. A. Mydosh.

*On leave from the Institute of Physics, Polish Academy of Sciences, Al. Lotnikow 32/46, 02-668 Warsawa, Poland.

${ }^{1}$ W. P. Lowe and T. H. Geballe, Phys. Rev. B 29, 4961 (1984).

2 J. M. Triscone, D. Ariosa, M. G. Karkut, and O. Fischer, Phys. Rev. B 35, 3238 (1987).

${ }^{3}$ I. Banerjee, Q. S. Yang, C. M. Falco, and I. K. Schuller, Solid State Commun. 41, 805 (1982).

${ }^{4}$ P. R. Auvil and J. B. Ketterson, Solid State Commun. 67, 1003 (1988).

${ }^{5}$ N. Missert and M. R. Beasly, Phys. Rev. Lett. 63, 672 (1989).
${ }^{6}$ M. Ikebe, Y. Muto, S. Ikeda, H. Fujimori, and K. Suzuki, Physica 107B, 387 (1981).

${ }^{7}$ N. R. Werthamer, Phys. Rev. 132, 2440 (1963).

${ }^{8}$ P. G. de Gennes, Rev. Mod. Phys. 36, 225 (1964).

${ }^{9}$ S. Maekawa and H. Fukuyama, J. Phys. Soc. Jpn. 51, 1380 (1981).

${ }^{10}$ J. M. Graybeal and M. R. Beasly, Phys. Rev. B 29, 4167 (1984).

${ }^{11}$ B. Y. Yin and J. B. Ketterson, Adv. Phys. 38, 189 (1989). 